\title{
Urban Intersections and Traffic Safety in the City of San Antonio
}

\author{
Qasim Adegbite, Khondoker Billah*, Hatim Sharif, and Samer Dessouky \\ Department of Civil and Environmental Engineering, University of Texas at San Antonio, San Antonio, TX 78249
}

\begin{abstract}
Intersections are high-risk locations on roadways and often experience high incidence of crashes. Better understanding of the factors contributing to crashes and deaths at intersections is crucial. This study analyzed the factors related to crash incidence and crash severity at intersections in San Antonio for crashes from 2013 to 2017 and identified hotspot locations based on crash frequency and crash rates. Binary logistic regression model was considered for the analysis using crash severity as the response variable. Factors found to be significantly associated with the severity of intersection crashes include age of driver, day of the week, month, road alignment, and traffic control system. The crashes occurred predominantly in the highdensity center of the city (downtown area). Overall, the identification of risk factors and their impact on crash severity would be helpful for road safety policymakers to develop proactive mitigation plans to reduce the frequency and severity of intersection crashes.
\end{abstract}

\section{Introduction}

Road intersections are one of the most dangerous locations in a roadway network as they are the convergent spots for vehicles and people moving in opposite paths. They often impose a major responsibility on road users to make an informed decision on when to cross the road to forestall unsafe situations. This part of the road has one of the most complicated traffic situations that motorists come across. Negligent high speeding drivers who often disregard traffic signals further compounds the problem [1]. In the United States, about one-quarter of road fatalities and about half of all traffic injuries over the last several years are attributed to intersections [2]. Intersection crashes account for more than $40 \%$ of all crashes in the United States [3]. A study examining the characteristics of 787,236 motor vehicle intersection crashes from 2005 through 2007, as listed in the dataset from the National Motor Vehicle Crash Causation Survey [4], showed that $96 \%$ of intersection crashes had critical factors attributed to drivers and less than $3 \%$ had critical factors were related to the vehicle or environment [4]. Ensuring safety of road intersections is a complicated issue and cannot always be mitigated by barely changing/adding signals and signs. A more sustainable solution can be achieved by a national comprehensive program of improved pedestrian safety and vehicle intersection management [4]. Proper identification of intersections associated with high crash rate is vital to reduce future crash occurrence. As of 2016, Texas has the highest number of fatal crashes in the United States; 3,406 crashes resulting in 3,776 deaths in that year. This is over 5\% increase over 2015 figures of 3,226 fatality crashes and 3,582 deaths. There was also a $5.09 \%$ increase in total miles traveled in 2016 (271.263 billion) compared to 2015 (258.122 billion).
Over 800 of these deaths occurred at intersections or were related to an intersection. Bexar County, where San Antonio is located, had the 3rd highest totals in Texas in 2016, with 52,633 crashes and 220 deaths because of the crashes [5]. Several factors contribute to intersection crashes including driver related factors, vehicle related factors, environment and road-related factors. Driver errors are mostly human related factors which include those committed while under the influence of alcohol, or otherwise violating traffic rules and regulations, and errors committed due to lack of training. Road-related factors include traffic characteristics e.g. traffic volume, geometric design of the intersections, traffic control measures, state of line markings and signage, and surface and light condition [6]. Environment-related factors, such as weather conditions and natural lighting conditions are mostly considered external to human factors as they are beyond human control. Another identified problem is the difficulty of many drivers in judging gaps in high-speed traffic situation on a divided highway with many lanes, as well as drivers trying to do left-turn or cross into a divided highway [7]. Human factors are the main cause of over $90 \%$ of car crash incidents [8].

The viewpoint of science of safety has shifted due to the evolution of data science. The focus is now on data and analysis rather than sole dependence on standards as stated in FHWA's Highway Safety Improvement Program Manual. For data analysis and safety consideration, three to five years of crash data is the most common time frame for good data representation. Less than five years data might be good enough for some cases. But a smaller sample size does not adequately represent the data in general and might require statistical adjustment [9]. The need for field assessment of intersection spots has been identified as a major activity

* Corresponding author: khondoker.billah@utsa.edu 
that can ensure the further safety of intersections. This will help with knowledge of drivers' behaviors and which of them needs improvement and periodic assessment [10]. The main purpose of the project was to understand the relationship between road network characteristics and traffic safety with a focus on intersections. The results obtained will provide valuable information to the City of San Antonio, Texas Department of Transportation, and other stakeholders in developing the right mitigation improvements for severe and fatal intersection crashes.

\section{Data and methodology}

The crash data for the period of January 2013 to December 2017 were provided by the Texas Department of Transportation's Crash Records Information System (CRIS) for use in this study. A logistic regression model was developed to test the relationship of crash severity with other crash associated factors. To develop the logistic model, 10 factors judged as possibly most relevant to intersection crash causality were selected from the data set according to literature review and previous similar studies. The selected variables are: age of driver, gender of driver, ethnicity of driver, time of day, day of week, month of year, lighting condition, weather condition, traffic control type, and road alignment. Crash severity was the response variable, in which death and incapacitating injury causing crashes were classified as "severe" and rest were classified as "non-severe". In this paper, the logit was the natural logarithm of the odds that the response variable $\mathrm{Y}$ was severe $(\mathrm{Y}=1)$ versus non-severe $(\mathrm{Y}=0)$, as shown by Equation (1):

$$
\operatorname{Logit}(\mathrm{P})=\operatorname{In}(\mathrm{P} / 1-\mathrm{P})=\beta_{0}+\beta_{1} * \mathrm{X}_{1}+\ldots .+\beta_{\mathrm{i}} * \mathrm{X}_{\mathrm{i}}
$$

Where $\mathrm{P}$ is the probability of severe crashes, $\mathrm{X}_{\mathrm{i}}$ is the independent variable, and $\beta_{\mathrm{i}}$ is the model coefficient directly determining the odds ratio. Among several available factors, crash severity, age and gender of driver, time of day, day of week, month of year, lighting condition, and weather condition were chosen for further analyses. Geospatial data was analyzed by crash locations (points) to evaluate the geospatial distribution of crashes and areas with higher densities of occurrences (hotpots). Intersections with high-volume crashes during the 5-year period under study were identified and the intersection crash rates for these high-volume crash intersections were calculated. These intersections were reclassified based on the crash rate and mapped. ArcGIS and computer software RStudio were used for data processing, analysis, and visualization.

\section{Results and discussion}

The logistic regression found age of driver, traffic control ID (crosswalk, yield sign), road alignment (straight-level, straight-grade), day of week (specially Sunday), and crash month (December) to be more significant for crash severity. Dark lighting condition and late hours at night (1 a.m.-4 a.m.) had smaller pvalues compared to their counterparts. Effect of road alignment and traffic control system on crash severity will be carried out in later studies. A total of 73,755 intersection crashes occurred in San Antonio during the study period and a total 145,263 primary persons (drivers of motor vehicle or motorcycle, bicyclists, and pedestrians) were related with these crashes. Among the primary persons, 141,627 were drivers of motor vehicles; 813 were bicyclists; 1,471 were pedestrians; 980 were driver of motorcycle type vehicles; and the rest were classified as either not reported or unknown. The number of intersection crashes, as well as total crashes in San Antonio shows an increasing trend from 2013 through 2016 while there was a slight downward trend in 2017, possibly due to some interventions that the city has implemented on some of the roads. The average percentage of intersection crashes to total crashes over the five years was around $29 \%$ as shown in the secondary $\mathrm{Y}$-axis in Figure 1. For the variable crash severity, the categories are incapacitating-injury (A), non-incapacitating injury (B), possible injury (C), killed $(\mathrm{K})$, and not injured or property damage only (PDO). The average percentage of $\mathrm{K}$ and $\mathrm{A}$ crashes in all the intersection crashes over the 5-year period was about $2.2 \%$, while $23 \%$ of the crashes resulted in possible injury. Intersection related crashes include 155 fatal crashes $(20.2 \%$ of all fatal crashes in San Antonio) and 1,447 serious injury crashes $(36.2 \%$ of serious injury crashes) from 2013-2017. The yearly variation of K\&A crashes and percentage of K\&A crashes to total crashes is shown in Figure 2. Percentage of K\&A crash was comparatively high in 2013. The number of fatal and incapacitating crashes by year does not show a clear pattern.

The volume of traffic flow and the frequency of intersection crashes vary with of the day and higher traffic volumes coincide with higher frequencies of intersection crashes. Figure 3 shows the hourly distribution of intersection crashes and percentage of intersection crashes in total crashes. Crash frequency is high from 12 p.m. - 7 p.m. with the peak during 5p.m. 6 p.m. Although intersection crash frequency from 7 p.m. - 11 p.m. is moderate, their percentage of total crashes is higher during this period. Both intersection crash frequency and their percentage in total crashes are considerably low from 1 a.m. - 6 a.m. This study also tried to explore the hourly variation of intersection crashes in different seasons. Hourly variation based on day of week (weekend or weekday) was also explored. Time of day is divided in total eight 3-hour intervals starting at midnight. The months are divided in four seasons: winter (January, February, and the following December), spring (March-May), summer (JuneAugust), and fall (September-November). For all seasons, 3:00 p.m. - 6:00 p.m. period had highest percentage of intersection crashes followed by the 12:00 p.m. - 3:00 p.m. period (Figure 4). The summer months had higher percentage of intersection crashes around afternoon; especially from 12:00 p.m. - 3:00 p.m.; compared to other seasons. This can be attributed to the distraction and discomfort resulting from excessive heat. 
Summer months also had noticeably lower intersection crash percentage from 6:00 a.m. - 9:00 a.m. which might be related to summer not being a school season. Intersection crash percentage was comparatively low from 6:00 p.m. - 9:00 p.m. during spring and from 9:00 p.m. - 12:00 a.m. during winter and fall. All 3-hour time segments had greater number of weekday intersection crashes than weekend intersection crashes except for 12:00 a.m. - 3:00 a.m. period (Figure 5). Higher number of traffic flow and higher number of drunk drivers at late night during weekend might be contributing to this anomaly. 6:00 a.m. - 9:00 a.m. period during weekend had very lower concentration of intersection crashes.

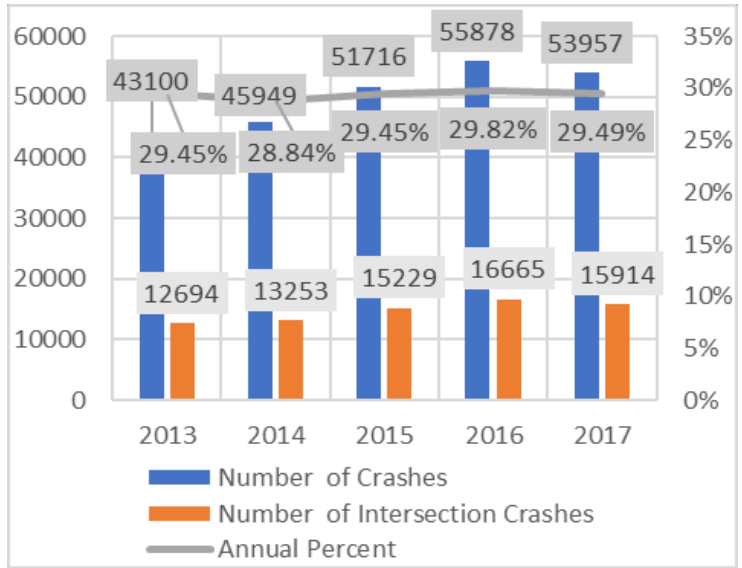

Fig. 1. Average $\%$ of intersection crashes in total crashes.

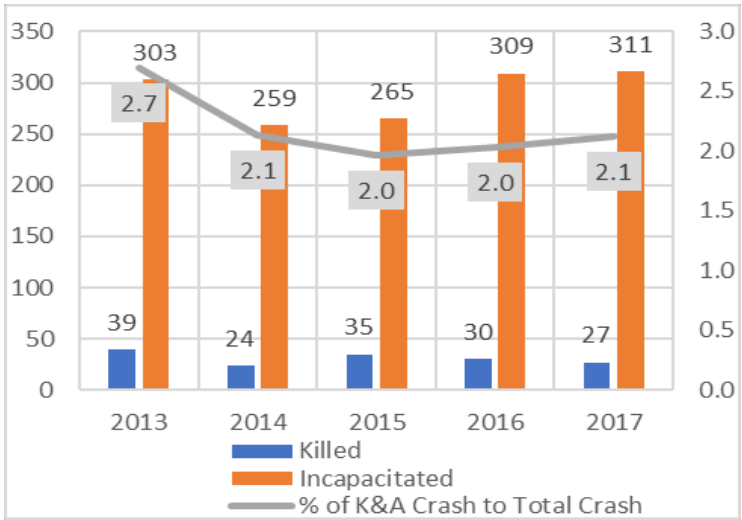

Fig. 2. Yearly variation of K\&A crashes and $\%$ of K\&A crashes in total crashes.

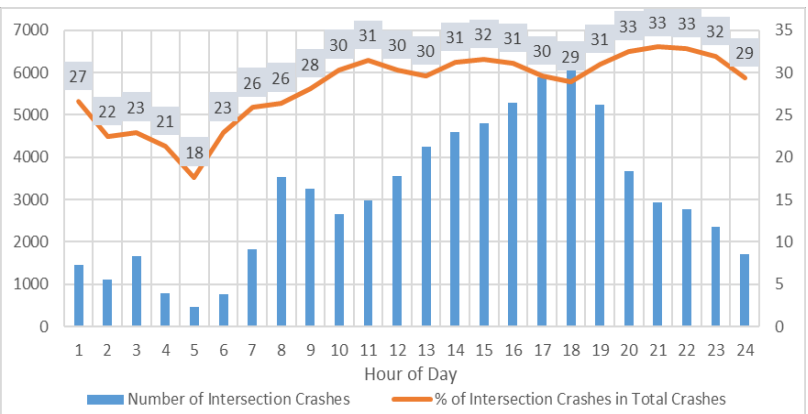

Fig. 3. Hourly distribution of intersection crashes and $\%$ of total crashes.

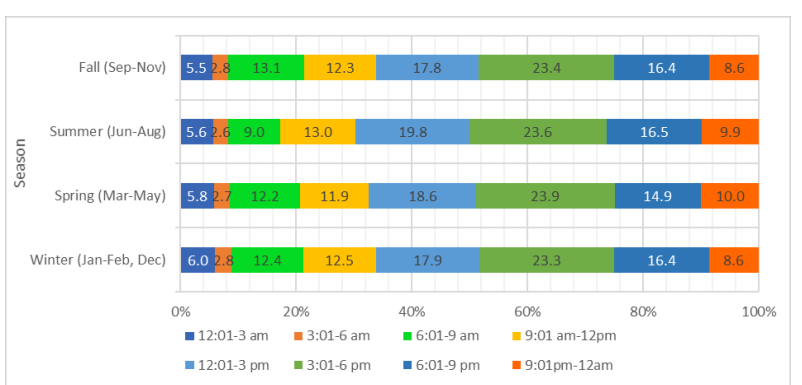

Fig. 4. Hourly variation of intersection crashes by season.

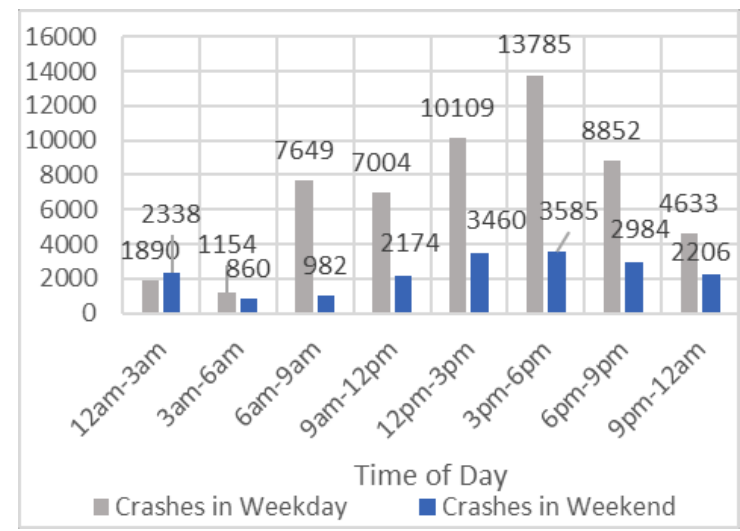

Fig. 5. Hourly variation of intersection crashes by day of the week.

The susceptibility to crashes is influenced by age and gender of drivers [11]. A total of 141,627 motor vehicle drivers were associated with intersection crashes. The age of 17,320 drivers were unavailable. Among the drivers with available age, the gender of 102 drivers were unavailable. All the drivers associated with intersection crashes with known age and gender were classified in eight age groups. There were 38 incidents when the driver was aged 14 or less. $26(68 \%)$ of those incidents had male drivers. Approximately $48 \%$ of the crashes happened with drivers in the age range of 15-34 years (Figure 6). The number of female licensed drivers is slightly greater than male licensed drivers in Texas [12]. The miles traveled by them might not be directly proportionate to their numbers. This study found increasing proportion of male drivers to female drivers related to intersection crashes over the age 44 . The ratio of male to female drivers for the age group 35-44 was closest to 1 but still number of male drivers was higher. For any day of the week, the percentage of intersection crashes in total crashes varied from approximately $29 \%$ to $30 \%$ (Figure 7). Friday had the highest number of intersection crashes. Sunday had considerably lower frequency of intersection crashes but the highest number fatal intersection crashes (Figure 7 and Figure 8). The percentage of K\&A crashes to total intersection crashes was highest for Sunday. Wednesday had highest number of incapacitating injury crashes. Overall, the frequency of fatal crashes and percentage of K\&A crashes in total intersection crashes were higher during the weekend (Figure 8). K\&A crash frequency at intersections was lower during winter and higher during fall (Figure 9). Percentage of K\&A intersection crashes to total intersection crashes was comparatively high during fall. 
The late summer, fall months, and early winter (AugDec) had considerably higher number of fatal crashes.

Dark conditions were associated with higher percentage of fatal crashes (almost 4 times) and serious injury crashes compared to "Daylight" lighting condition (Figure 10). This might be attributed to the impaired visibility during late hours of night due to lesser traffic volume. Drivers might be tempted to break laws at late hours due to lesser traffic at intersections. Dark conditions (both lighted and not lighted) had higher percentage of pedestrian crash during weekend (Figure 11). Approximately 21\% intersection crashes occur under "Daylight" condition while "Dark, Not Lighted" and "Dark, Lighted" conditions are responsible for approximately $31 \%$ and $36 \%$ intersection crashes respectively during weekend. The percentage of both fatal and incapacitating injury crashes in total intersection crashes was lower during "Rain" weather condition (Figure 12). This might be attributed to the drivers being more cautious and driving at lower speeds due to lower visibility and surface friction.

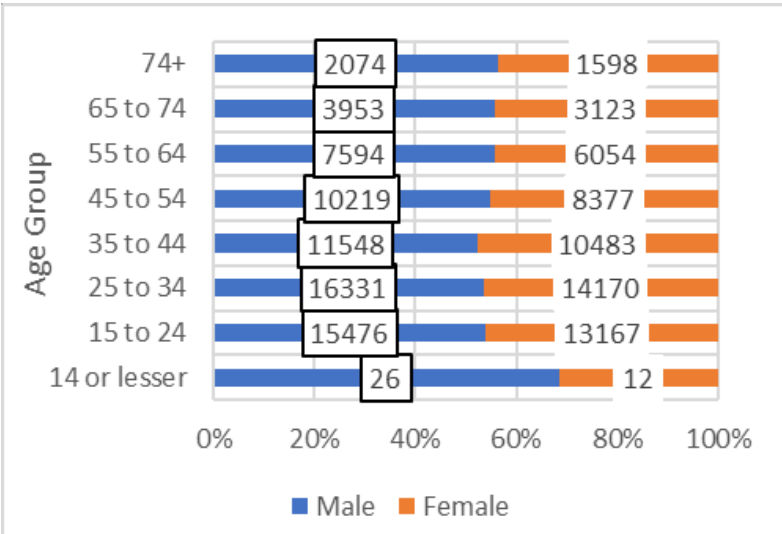

Fig. 6. Intersection crashes by age and gender.

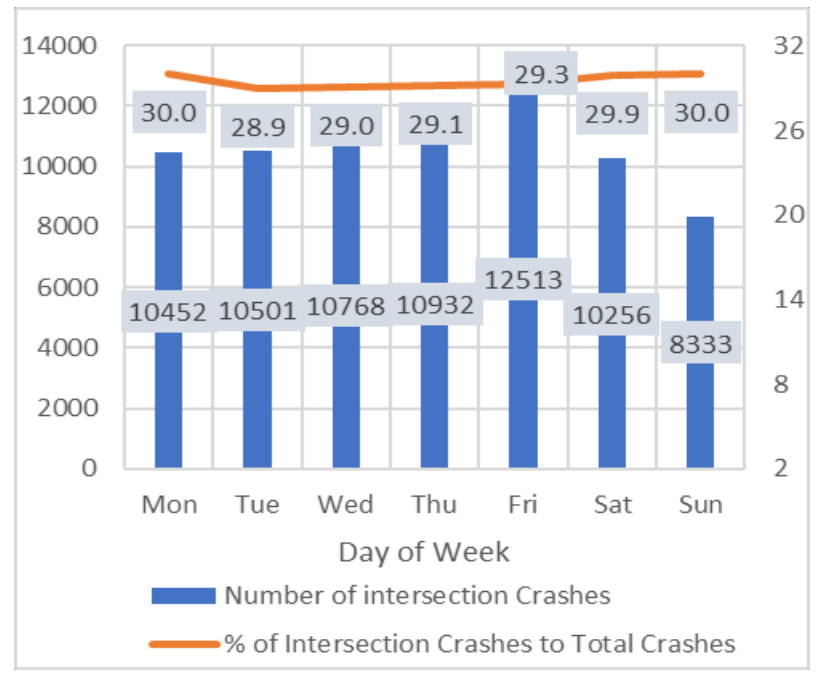

Fig. 7. Intersection crashes and $\%$ of intersection crashes in total crashes by day of week.

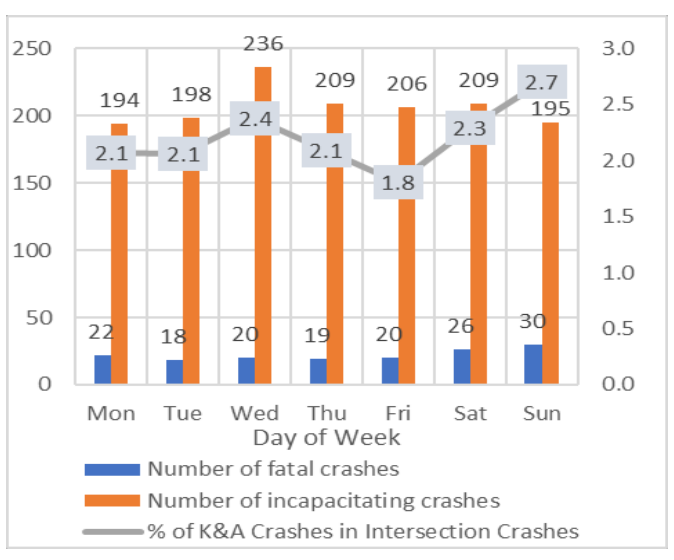

Fig. 8. The frequency of fatal crashes and percentage of K\&A crashes in total intersection crashes.

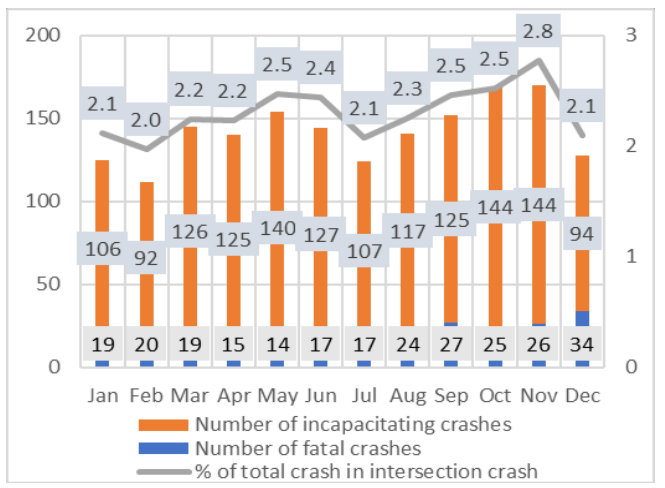

Fig. 9. Monthly K\&A crash frequency at intersections.

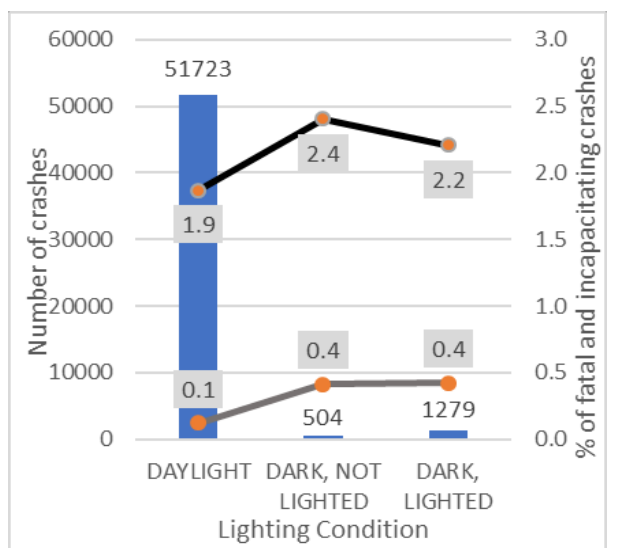

Fig. 10. Intersection crashes by light condition and $\% \mathrm{~K} \& \mathrm{~A}$ crash.

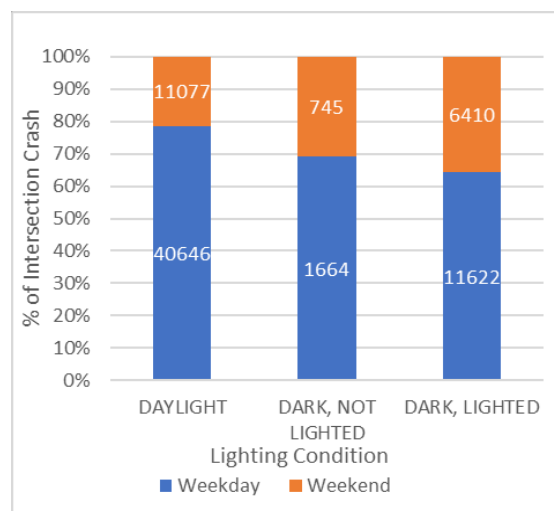

Fig. 11. Intersection crashes by light condition \& day of week. 


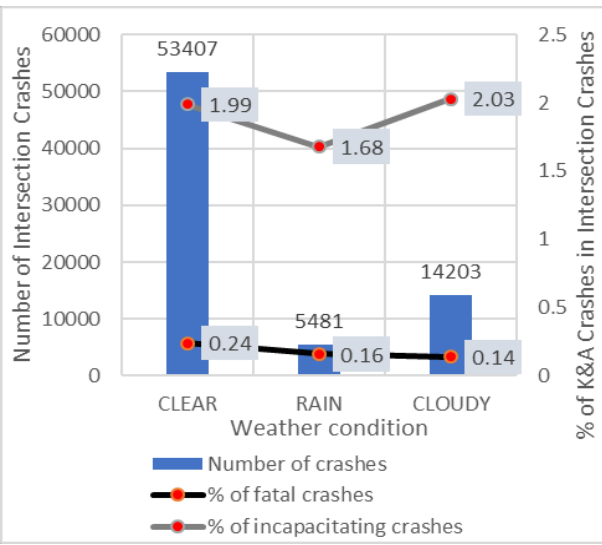

Fig. 12. Intersection crashes by weather condition.

All crash frequency data and the crash rate calculated based on AADT for each intersection were mapped to show the spatial distribution of high-risk intersections (Figure 13 and Figure 14). The results of the hotspot analysis were superimposed on the open street map of San Antonio for better visualization of hotspot locations. The most prominent hotspot intersections are in the central, west, northwest and northeast side of San Antonio (Figure 15).

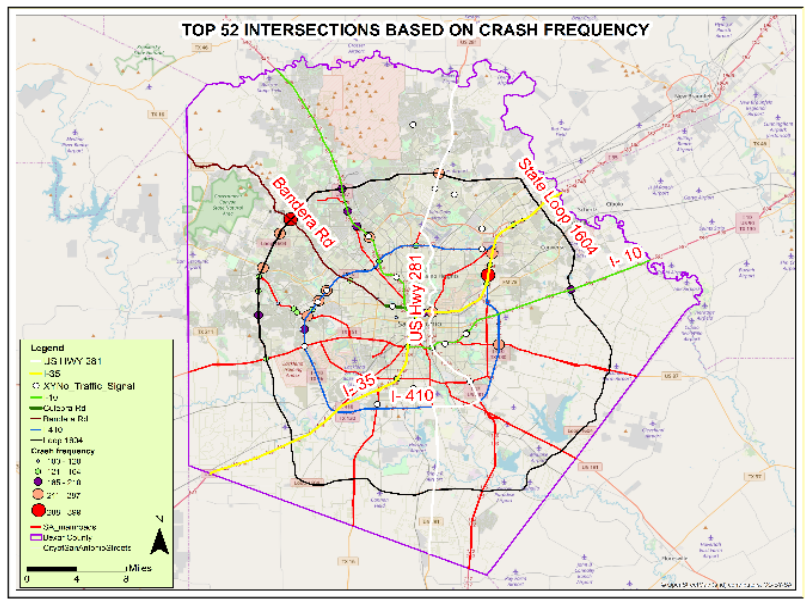

Fig. 13. Top 52 intersections based on crash frequency.

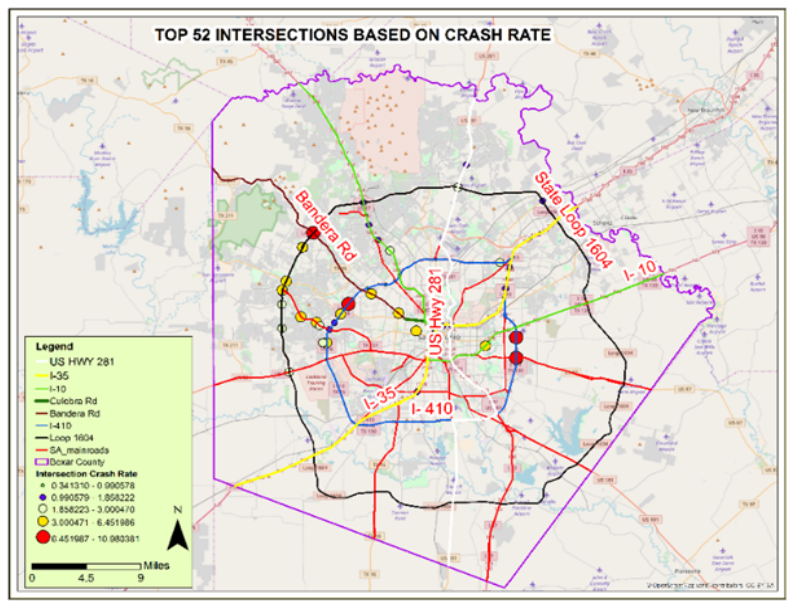

Fig. 14. Top 52 intersections based on crash rate.

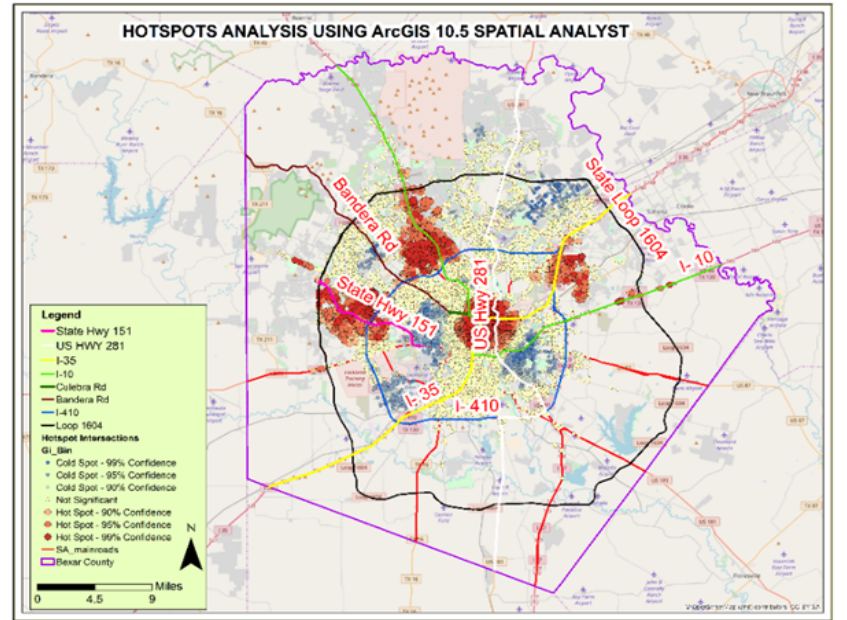

Fig. 15. Map of hotspot analysis using ArcGIS spatial analyst tool.

\section{Conclusions}

To show the factors that influence severity of intersection crashes, a logistic regression method was applied to a set of traffic collision data obtained from CRIS. Age of driver, traffic control system, alignment of road, day of week, and month of occurrence were found to be statistically significant. Results show that younger drivers between the ages of 15 and 34 years old are at greater risk of being involved in crashes. Higher percentage of fatal crashes and serious injuries intersection crashes were associated with dark conditions which can be due to the tendency of over-speeding and disregarding signals at late hours with lesser traffic volume. Over $60 \%$ of the crashes occurred under clear weather condition which is a pattern suited for the novel self-driving technology which can handle simple, monotonous tasks more proficiently and effectively than human beings. Driver inattention and disregard for traffic signal respectively represented more than half of the reported contributing factors to intersection crashes. Effect of road alignment and traffic control system on severe crashes requires further analyses. The hotspot analysis showed that intersection crashes occurred predominantly around the city center having higher concentration of intersections and traffic. Further research on the geospatial relationship and empirical studies among different risk factors are important to provide more appropriate countermeasures to reduce fatality and serious injuries to zero.

\section{References}

1. United States Department of Transportation, Federal Highway Administration. MUTCD 2003 manual on uniform traffic control devices. [Washington, D.C.].

2. NHTSA, 2010b. Crash Factors in IntersectionRelated Crashes: An On-Scene Perspective: DOT HS 811 366. National Highway Traffic Safety Administration, Washington, DC (September 2010).

3. Lord, D., Schalkwyk, I., Chrysler, S., and Staplin, L. (2007). A Strategy to Reduce Older Driver Injuries at 
Intersections Using More Accommodating Roundabout Design Practices. Accident Analysis \& $\begin{array}{llll}\text { Prevention } & 39 & \text { (3): }\end{array}$ https://doi.org/10.1016/j.aap.2006.09.011.

4. Singh, S. (2015). Critical reasons for crashes investigated in the National Motor Vehicle Crash Causation Survey. (Traffic Safety Facts Crash Stats. Report No. DOT HS 812 115). Washington, DC: National Highway Traffic Safety Administration.

5. Texas Motor Vehicle Traffic Crash Facts http://ftp.dot.state.tx.us/pub/txdotinfo/trf/crash_statistics/2016/01.pdf

6. Hussein. N., and Hassan, R. (2017). Surface Condition and Safety at Signalised Intersections. International Journal of Pavement Engineering 18 (11): 1016-26. https://doi.org/10.1080/10298436.2016.1141411.

7. Burchett, G., and Maze, T. (2005). Rural Expressway Intersections that Contribute to Reduced Safety Performance. Proc., 2005 MidContinent Transportation Research Symposium, Iowa State University, Ames, Iowa.

8. Federal Highway Administration. (2009). Intersection Safety Issue Briefs. FHWA Safety. http://safety.fhwa.dot.gov/intersection/resources/\#fh wasa10005 (Accessed December 21, 2017)

9. Persaud, B.N. (2001). Statistical Methods in Highway Safety Analysis. NCHRP Synthesis 295. Transportation Research Board, Washington, DC.

10. Smeed, R.J. (1974). Research into driver behaviour. Meeting of the International Statistics Institute. Vienna, Austria.

11. Chen, H., Cao, L., and Logan, D.B. (2012). Analysis of Risk Factors Affecting the Severity of Intersection Crashes by Logistic Regression. Traffic Injury Prevention, 13(3), 300-307. https://doi.org/10.1080/15389588.2011.653841.

12. Table DL-22 - Highway Statistics 2016 - Policy | Federal Highway Administration. Accessed October 26, 2018. https://www.fhwa.dot.gov/policyinformation/statisti cs/2016/d122.cfm. 